\title{
Interfacial tension and wetting in colloid-polymer mixtures
}

\author{
D. G. A. L. Aarts, ${ }^{a}{ }^{2}$ R. P. A. Dullens, and H. N. W. Lekkerkerker \\ Van 't Hoff Laboratory for Physical and Colloid Chemistry, Debye Institute, University of Utrecht, \\ Padualaan 8, 3584 CH Utrecht, The Netherlands \\ D. Bonn \\ Laboratoire de Physique Statistique, Ecole Normale Supérieure, 24, Rue Lhomond, \\ 75231 Paris cedex 05, France, Van der Waals-Zeeman Instituut, Universiteit van Amsterdam, Valckenierstraat \\ 65, 1018 XE Amsterdam, The Netherlands \\ R. van Roij \\ Institute for Theoretical Physics, University of Utrecht, Leuvenlaan 4, 3584 CE Utrecht, The Netherlands
}

(Received 16 September 2003; accepted 29 October 2003)

\begin{abstract}
We calculate the interfacial tension and the wetting behavior in phase separated colloid-polymer mixtures both for ideal and excluded volume interacting polymers. Within the recently developed extension of the free volume theory to include polymer interactions [Aarts, Tuinier, and Lekkerkerker, J. Phys.: Condens. Matter 14, 7551 (2002)] the interfacial tension of the free interface is calculated by adding a van der Waals squared gradient term. The wetting behavior at a hard wall is calculated following a Cahn-Fisher-Nakanishi approach taking the one- and two-body colloid-wall interactions into account. Comparing results for interacting polymers with those for ideal polymers we find that for interacting polymers the interfacial tension does not increase as steeply as a function of the gas-liquid colloid density difference. Furthermore, the wetting transition shifts to higher polymer concentrations, even to above the triple line. The predictions for both the interfacial tension and the wetting are compared to recent experiments. (C) 2004 American Institute of Physics. [DOI: 10.1063/1.1635810]
\end{abstract}

\section{INTRODUCTION}

Mixtures of colloids and nonadsorbing polymer display rich phase behavior and are an excellent tool for studying equilibrium properties, phase transition kinetics, and metastable gel or glass states (for a recent comprehensive review, see Ref. 1). Recently, the interfacial tension between the coexisting phases received some attention, first experimentally by measurements of the ultralow interfacial tension of the order of at most a few $\mu \mathrm{N} / \mathrm{m}$ between demixed colloidal "liquid" (rich in colloid, poor in polymer) and colloidal "gas" (poor in colloid, rich in polymer) phases. ${ }^{2-4}$ This in turn led to a number of theoretical efforts, starting with a paper by Vrij. ${ }^{5}$ Within the Asakura-Oosawa-Vrij (AOV) model, ${ }^{6,7}$ which treats the polymers as ideal mutually penetrable hard spheres (PHS), Brader and Evans ${ }^{8}$ calculated the interfacial tension using a squared gradient approach. In the work of Brader and Evans the degrees of freedom of the polymer were integrated out such that an effective onecomponent system was obtained, similar but not equal to the semigrand potential derived using the free volume theory. ${ }^{9}$ The density inhomogeneities were taken into account by a squared gradient term. The order of magnitude of the resulting interfacial tensions compares well to the experimental values even though the predicted phase diagram does not quantitatively correspond to the experimental bulk phase dia-

a)Electronic mail: d.g.a.1.aarts@chem.uu.nl gram. The fact that the experimental bulk phase diagram is not well described by theory is that in experiment polymers behave far from ideal.

Theory preceded experiment in the prediction of the existence of a wetting transition of the mixtures in contact with a hard planar wall. ${ }^{10}$ At this wetting transition the liquid phase starts wetting the wall completely instead of partially. Again polymers were described as PHS but they were now explicitly described, hence the colloid-polymer mixture is treated as a true binary mixture, within the formalism of fundamental measures theory. ${ }^{11}$ For homogeneous phases this density functional reduces exactly to the aforementioned free volume theory. ${ }^{9}$ Moreover, it can be extended to a mixture in the vicinity of a hard wall. Doing so, in addition to layering transitions, a first-order wetting transition was found. ${ }^{10}$ These results were recently confirmed independently using computer simulations by Dijkstra and van Roij, ${ }^{12}$ again describing polymers as PHS. They found transitions reasonably close to those of the predictions in Ref. 10. First experiments did confirm that the colloidal liquid phase favors the wall and the accompanying interfacial tension was in good agreement with previous measurements. ${ }^{13}$ Whether or not the liquid phase was partially or completely wetting could not be concluded in this work, because of the difficulty in measuring the contact-angles with sufficient accuracy. Very recently, a wetting transition was reported in a similar colloid-polymer mixture. ${ }^{14,15}$ The authors mention the difficulty of comparing the experimentally found location of the wetting transition to the theoretical prediction. Again, 

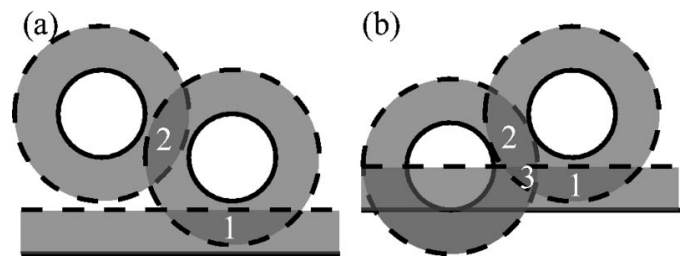

FIG. 1. Two possible configurations for two colloids near a flat hard wall. The depletion zones are indicated by dashed lines. In (a) we have a colloidwall interaction (1) and a colloid-colloid interaction (2) while in (b) we also have a colloid-colloid-wall interaction (3).

the reason for this is that in experiment polymers behave far from ideal. Several ways to describe polymers more realistically are available in literature ${ }^{16-23}$ mainly focusing on bulk phase behavior. Here we extend the earlier model of Ref. 17 for bulk to calculate the interfacial tension and to describe wetting behavior now also for interacting polymers.

That the colloidal liquid phase (partially) wets the wall is normally explained with "classical" AOV-like arguments, hence in terms of overlap volumes and accompanying interactions (see Fig. 1). Since at contact the overlap-volume between a wall and a colloid [volume 1 in Fig. 1(a)] is about twice the overlap-volume between two colloids [volume 2 in Fig. 1(a)] the colloidal liquid phase favors the wall, although the pair attraction is reduced if two colloids are close to the wall and to each other [volume 3 in Fig. 1(b)]. One of the goals of this work is to put this qualitative view in a quantitative form using the Cahn theory of wetting. ${ }^{24}$

We work at a semigrand canonical level, treating the polymers grand canonically and the colloids canonically. We thus have an effective one component system of colloids at a constant chemical potential of the polymer maintained by a reservoir filled with only polymer. We focus on predictions of the bulk phase behavior, the interfacial tension, the wetting transition and the prewetting line for ideal polymers as well as for excluded volume interacting (EVI) polymer chains. To keep descriptions simple and straightforward we describe the inhomogeneities with a squared gradient term as in Ref. 8, but with the free volume expression ${ }^{9}$ for the bulk free energy. The interaction with the wall is described up to second order in colloid contact density, i.e., we follow a Fisher-Nakanishi-type ${ }^{25}$ extension of the Cahn theory of wetting. ${ }^{24}$ (for a recent review see Ref. 26). We present theory for the bulk phase behavior in Sec. II, for the free interface in Sec. III and for the mixture near a flat hard wall in Sec. IV. We summarize our main findings in Sec. V.

\section{BULK PHASE BEHAVIOR}

The starting point of our analysis is the thermodynamic potential $F\left(N_{c}, V, T, \mu_{p}^{r}\right)$ of a bulk fluid of $N_{c}$ colloids in a volume $V$ and with temperature $T$, in osmotic contact with a polymer reservoir of chemical potential $\mu_{p}^{r}$ (and osmotic pressure П). Using the free volume approach of Ref. 9 yields

$$
F\left(N_{c}, V, T, \mu_{p}^{r}\right)=F_{0}(N, V, T)-V \int_{0}^{n_{p}^{r}} d n_{p}^{r \prime} \alpha\left(\frac{\partial \Pi}{\partial n_{p}^{r \prime}}\right) .
$$

In Ref. 17 we derived this in detail, showed how to make the extension from ideal to interacting polymer chains, and gave the necessary expressions explicitly. Here, we will only briefly repeat some of it. In (1) $F_{0}$ is the free energy of the pure hard sphere system, i.e., without added polymer, $\alpha$ is the free volume fraction, $\Pi$ the osmotic pressure, and $n_{p}^{r}$ the polymer number density in the reservoir. The polymer concentration in the system is given by: $n_{p}=\alpha n_{p}^{r}$. For $F_{0}$ we use the Carnahan-Starling equation of state ${ }^{27}$ to describe the fluid, gas and liquid phases and we make use of a reference free energy obtained from computer simulation $\mathrm{s}^{28}$ to describe the crystalline phase. The osmotic compressibility $\left(d \Pi / d n_{p}^{r}\right)$ depends on the nature of the polymers; for ideal polymers it equals $1 / \beta$ with $\beta=1 / k_{B} T$ and for polymers in the full excluded volume limit we use expressions from renormalization group theory [Eq. (17.53) from Ref. 29]

$$
\beta\left(\frac{d \Pi}{d n_{p}^{r}}\right)=1+2.629 \phi_{p}^{r}\left(\frac{1+3.251 \phi_{p}^{r}+4.151\left(\phi_{p}^{r}\right)^{2}}{1+1.480 \phi_{p}^{r}}\right)^{0.309},
$$

with $\phi_{p}^{r}=n_{p}^{r} v_{p}$ and $v_{p}=\frac{4}{3} \pi R_{g}^{3}\left(R_{g}\right.$ is the polymer's radius of gyration). The free volume fraction $\alpha$ is given by

$$
\alpha=(1-\phi) \exp \left[-\left(A d+B d^{2}+C d^{3}\right)\right],
$$

with $\phi$ the volume fraction of colloids and $d=\phi /(1-\phi)$. The parameters $A, B$, and $C$ are functions only of the depletion thickness (in this approach a step function with thickness $\Delta$ ) divided by the radius of the colloid $R_{c}$

$$
\begin{aligned}
& A=3 \frac{\Delta}{R_{c}}+3\left(\frac{\Delta}{R_{c}}\right)^{2}+\left(\frac{\Delta}{R_{c}}\right)^{3}, \\
& B=\frac{9}{2}\left(\frac{\Delta}{R_{c}}\right)^{2}+2\left(\frac{\Delta}{R_{c}}\right)^{3}, \\
& C=3\left(\frac{\Delta}{R_{c}}\right)^{3} .
\end{aligned}
$$

For ideal polymers within the PHS approach $\Delta$ simply equals $R_{g}$ and $\Delta / R_{c}$ becomes $q$, the polymer to colloid size ratio. For EVI-polymers we take both curvature (a polymer can wrap around a colloid) and concentration effects into account. The depletion thickness now depends on the size ratio $q$ and on the polymer concentration. We use results from Hanke et al $^{30}$ to incorporate the curvature dependence

$$
\frac{\Delta}{R_{c}}=\left(1+3 a\left(\frac{R_{g}}{R_{c}}\right)+3 b\left(\frac{R_{g}}{R_{c}}\right)^{2}-3 c\left(\frac{R_{g}}{R_{c}}\right)^{3}\right)^{1 / 3}-1,
$$

with analytical expressions for $a, b$, and $c$ approximately equal to $1.071,0.869$, and 0.040 , respectively. To further incorporate polymer concentration dependence we replace $R_{g}$ with the polymer bulk correlation length [Eq. (19.24) in Ref. 29] which is in line with the work of Joanny et al. ${ }^{31}$ Having all ingredients for (1) we apply common-tangent constructions (after dividing the free energy by $V$ to switch to free energy densities) and are thus able to find the coexisting densities ( $\rho_{L}$ and $\rho_{G}$ for liquid and gas). In Fig. 2 we show the bulk gas-liquid binodals both for ideal (dashed line) and EVI-polymers (full line) in polymer system concentration. We compare the theoretical predictions to the experimentally determined binodal (symbols) for a colloidpolymer mixture with $q=1.08$ of stearyl-coated silica 


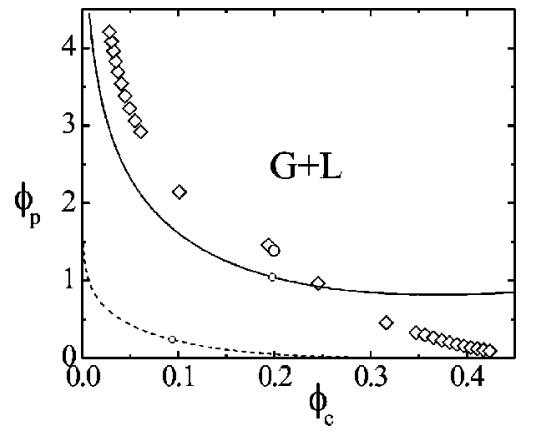

FIG. 2. Comparison of experimental phase diagram with theoretical predictions. The symbols denote the experimental binodal for a colloid-polymer mixture with size ratio $q=1.08$ (Ref. 3). The large open circle is the estimated critical point. The dashed line is the predicted binodal describing the polymers as penetrable hard spheres, while describing the polymers as having excluded volume interactions results in the full line. Open circles denote critical points.

colloids $\left(R_{c}=13 \mathrm{~nm}\right)$ with poly (dimethylsiloxane) polymer $\left(R_{g}=14 \mathrm{~nm}\right)$ in cyclohexane. ${ }^{3}$ Note that we have not shown crystal-fluid coexistence in this phase diagram. Experimentally, no crystal-fluid coexistence is found for any colloidpolymer concentrations, possibly because colloidal sphere polydispersity supresses crystallization. The predicted binodal for the mixture with ideal polymers does not agree quantitatively to the experimental binodal. The binodal for EVI-polymers shifts in the right direction, although it clearly predicts too high polymer concentrations above a certain colloid volume fraction, the reason for which is unknown. The overall agreement, however, has improved significantly and such a more quantitative agreement between the predicted phase behavior for mixtures with EVI-polymers compared to experimental systems is a first step in making quantitative predictions about surface tension and wetting phenomena.

\section{INTERFACE}

To describe the free colloidal liquid-gas interface we start with the functional for the surface tension $\gamma[\rho]$

$$
\gamma[\rho]=\int_{-\infty}^{\infty} d z\left[f(\rho)-\mu_{c} \rho+p_{c}+m\left(\frac{d \rho}{d z}\right)^{2}\right] .
$$

Here, $z$ is the distance to the interface, $f(\rho)=F / V$ is the free energy density, for which we use the semigrand canonical potential $F$ defined in (1) and divide by the volume $V$. Together with the second and third term ( $\mu_{c}$ being the chemical potential and $p_{c}$ the pressure at coexistence) this gives the excess free energy in the interfacial region. The last term in the integral accounts for density inhomogeneities. This quadratic term with coefficient $m$ is in fact the first term of an expansion in derivatives of the density.

The coefficient $m$ is given by the second moment of the direct correlation function $c(r)$ with $r$ the center-to-center distance and reads

$$
m \beta=\frac{\pi}{3} \int_{0}^{\infty} d r r^{4} c(r) .
$$

Here, we use the mean spherical approximation for the direct correlation function which only depends on the attractive

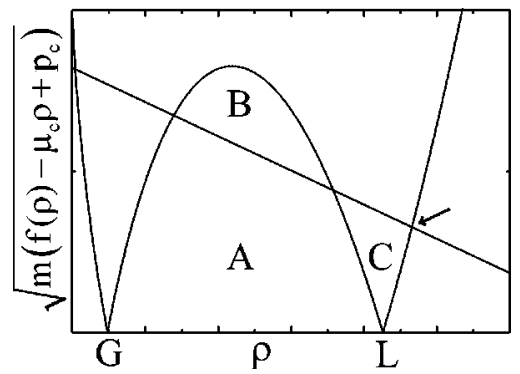

FIG. 3. Ignoring the straight line the area $\mathrm{A}+\mathrm{B}$ is proportional to the surface tension following from (12). The two minima are the gas-liquid coexistence points. Adding the straight line, which is given by the 1.h.s. of (23), enables one to solve (23) graphically. At the wetting transition the areas B and $\mathrm{C}$ must be equal. The arrow indicates the density of liquid phase at the wall.

part of the pair potential $u(r)$ and hence $c(r)=0$ for $r<\sigma_{c}$ (with $\sigma_{c}$ the colloid diameter) and $c(r)=-\beta u(r)$ for $r$ $\geqslant \sigma_{c}$. For ideal polymers the pair potential can be written as

$$
u(r)=-k_{b} \operatorname{Tn}_{p}^{r} V_{o}(r)
$$

with $n_{p}^{r}$ the polymer concentration and $V_{o}(r)$ the overlap volume between the two depletion zones. For general $q$ and ideal polymers modeled as PHS $m$ becomes

$$
\frac{m \beta}{\sigma_{c}^{5}}=\frac{\pi}{6} \phi_{p}^{r}\left[1+\frac{7 q}{4}+\frac{7 q^{2}}{5}+\frac{7 q^{3}}{10}+\frac{q^{4}}{5}+\frac{q^{5}}{40}\right] .
$$

For EVI-polymers the pair potential can be written as ${ }^{32}$

$$
u(r)=-\int_{0}^{n_{p}^{r}} d n_{p}^{\prime}\left(\frac{\partial \Pi}{\partial n_{p}^{\prime}}\right) V_{o}\left(r, n_{p}^{\prime}\right),
$$

and $m$ can be calculated numerically.

More sophisticated expressions for $c(r)$ could have been used, for example, by rewriting the direct correlation functions from the density functional in Ref. 11 to an effective colloid-colloid direct correlation function. However, the resulting $m$ can then become negative at high colloid densities, where the repulsive hard sphere contribution to $c(r)$ becomes more important than the attractive depletion contribution. This shows that the expansion in (6) in terms of gradients in the density does not converge.

Minimizing the interfacial tension (6) with respect to $\rho$ using functional differentiation leads to

$$
2 m \frac{d^{2} \rho}{d z^{2}}=\frac{d f(\rho)}{d \rho}-\mu_{c},
$$

which can be used to calculate the equilibrium interfacial tension (without actually having to know the true shape of the interfacial profile)

$$
\gamma=2 \int_{\rho_{G}}^{\rho_{L}} d \rho \sqrt{m\left(f(\rho)-\mu_{c} \rho+p_{c}\right)} .
$$

The interfacial tension is thus equal to area $A+B$ in Fig. 3 (ignoring the straight line whose meaning will be explained in Sec. IV).

In Fig. 4 we present the surface tension obtained from the functional in (6) for ideal polymers with $q=1.0$ (dashed line) and for EVI-polymers with $q=1.08$ (full line). We 


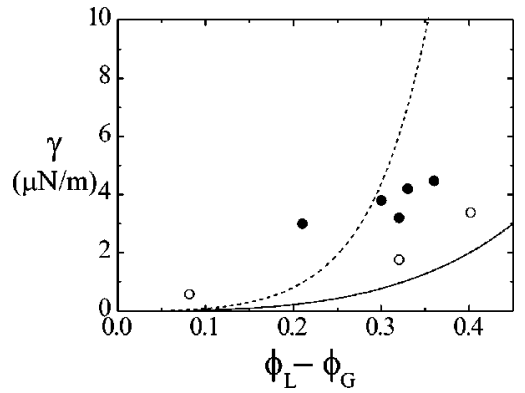

FIG. 4. Comparison of experimental surface tension with theoretical predictions. The circles denote surface tensions measured in a colloid-polymer mixture with size ratio $q=1.08$ of which the phase diagram is given in Fig. 2. Closed circles represent measurents done by De Hoog and Lekkerkerker using a spinning drop technique (Ref. 3) and open circles denote measurements by Aarts et al. obtained by analyzing the static profile near a vertical hard wall (Ref. 13) on exactly the same experimental system. The dashed line is the prediction describing the polymers as ideal, while describing the polymers as having excluded volume interactions results in the full line.

chose $q=1.0$ for ideal polymers in order to compare our results with existing theories ${ }^{8,10}$ and simulations ${ }^{12}$ and we chose $q=1.08$ for EVI-polymers to compare with experimental results. ${ }^{3,13}$ The change from $q=1.0-1.08$ leads to better agreement with experiment, while this change has no serious consequences for the comparison with theory. Also shown are experimental interfacial tensions determined by De Hoog and Lekkerkerker with a spinning drop apparatus for colloid-polymer mixtures at $q=1.08^{3}$ (filled circles). Aarts et al. measured the interfacial tension in exactly the same system using a different (static) technique ${ }^{13}$ (open circles): The gas-liquid interface was analyzed near a vertical hard wall, where the interface curvature depends only on the capillary length; from this the interfacial tension was obtained. The discrepancy between the two experiments shows the difficulty of measuring such ultra-low interfacial tensions. Clearly, our prediction for the interfacial tension for ideal polymers (PHS) rises rather fast compared to the experiment, which is not surprising at all, because there is no good agreement between the predicted phase diagram and the experimental phase diagram as was already mentioned in Sec. I and shown in Sec. II. The plot does illustrate, however, clear agreement with predictions by Brader et al. ${ }^{10}$ where fundamental measures theory was used to describe the colloid-polymer mixture. This agreement justifies the use of squared gradient theory. Furthermore, we would like to stress the importance of the bulk free-energy density on the predicted interfacial tensions, which is best illustrated by comparing our predictions to those from Brader and Evans. ${ }^{8}$ We describe the density inhomogeneities in the same way as in Ref. 8 , but use a different free-energy density. ${ }^{9}$ As a result, our predictions do not agree with those of Ref. 8, but do agree with the more sophisticated approach of Ref. 10.

For EVI-polymers the predicted interfacial tension slightly underestimates the experimental data points, although the data taken from Ref. 13 are reasonably followed. The smaller interfacial tensions as well as the shift in the binodal to higher polymer concentrations as shown in Fig. 2 means that in the description with EVI-polymers instead of ideal polymers the effect of shrinking depletion zones as a function of polymer concentration, which leads to smaller overlap volumes and hence smaller attractions, is more important than the steeply increasing osmotic compressibility as a function of polymer concentration, which leads to stronger attractions.

\section{COLLOID-POLYMER MIXTURE NEAR A HARD WALL}

The next step is to describe the colloid-polymer mixture near a hard wall. We use the same expressions as above and add the following terms to the functional in (6) (in which the integration now ranges from 0 to $\infty$ ) to incorporate the interactions with the wall

$$
\begin{aligned}
\gamma[\rho]= & \int_{0}^{\infty} d z\left[f(\rho)-\mu_{c} \rho+p_{c}+m\left(\frac{d \rho}{d z}\right)^{2}\right] \\
& +\int_{0}^{\infty} d z \rho(z) U_{2}(z) \\
& -\frac{1}{2} \int_{0}^{\infty} d z \int d \mathbf{r} \rho(z) \rho(\mathbf{r}) U_{3}(z, \mathbf{r})+\cdots .
\end{aligned}
$$

Here, $U_{2}$ is the attractive colloid-wall interaction, $U_{2} \leqslant 0$, and can be found from (10), in which the overlap volume $V_{o}$ now depends on the depletion zones of the wall and the colloid. The next term in (13) is a wall-induced correction (hence the minus sign) to the pair-wise colloid-colloid interaction, which is reduced [compared to its bulk pair interaction $u(r)]$ in the vicinity of the hard wall. This is illustrated in Fig. 1(b), where the volume labeled " 3 " is double counted [it contributes to $u(r)$ and $U_{2}(z)$ ] and should, therefore, be subtracted in order to give a proper account of the free volume. In other words, the system gains free volume only once. This is in fact the first-order correction since three and more particle interactions with the wall are also possible and become more important for large $q$. One can easily show that for ideal polymers there are no volumes 3 when $q$ becomes less than $1 / 4$. Thus this second term with $U_{3}$ depends on overlap volumes of type 3 [see Fig. 1(b)] to be included in (10) as $V_{o}$. We now should do this for all positions of the two particles with respect to each other and to the wall.

Instead of using the explicit form of (13) we make the following approximations:

$$
\int_{0}^{\infty} d z \rho(z) U_{2}(z) \approx \rho_{1} \int_{0}^{\infty} d z U_{2}(z) \equiv-\rho_{1} h_{1},
$$

in which we define the contact density as $\rho_{1} \equiv \rho(0)$ and $h_{1}$ $=-\int_{0}^{\infty} d z U_{2}(z)$. In addition we approximate

$$
\begin{gathered}
-\int_{0}^{\infty} d z \int d \mathbf{r} \rho(z) \rho(\mathbf{r}) U_{3}(z, \mathbf{r}) \\
\approx-\rho_{1}^{2} \int_{0}^{\infty} d z \int d \mathbf{r} U_{3}(z, \mathbf{r}) \\
\equiv \rho_{1}^{2} g
\end{gathered}
$$

with $g=-\int_{0}^{\infty} d z \int d \mathbf{r} U_{3}(z, \mathbf{r})$. Here we make the same approximation as in (14) and write the correlated density- 


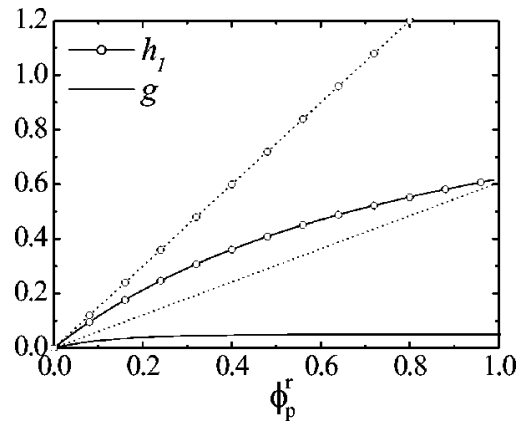

FIG. 5. The parameters $h_{1}$ (with symbol) in units of $\sigma_{c} / \beta$ and $g$ (without symbol) in units of $\sigma_{c}^{4} / \beta$ for $q=1.0$ as a function of polymer concentration for ideal (dotted) and EVI-polymers (full).

density product as the density squared. With these approximations we obtain a Cahn-Fisher-Nakanishi-type functional $^{24,25}$ in which the interaction with the wall is described up to second order in density with clear physical parameters $h_{1}$ and $g$. These are similar to the "surface chemical potential" and the "surface enhancement parameter" as in Ref. 25. Note that the arguments of the integrals vanish beyond a certain small distance to the wall, since the overlap volumes with the wall needed to calculate $U_{2}$ and $U_{3}$ rapidly decay to zero. Our working functional therefore reads

$$
\begin{aligned}
\gamma[\rho]= & \int_{-\infty}^{\infty} d z\left[f(\rho)-\mu_{c} \rho+p_{c}+m\left(\frac{d \rho}{d z}\right)^{2}\right] \\
& -\rho_{1} h_{1}+\frac{1}{2} \rho_{1}^{2} g .
\end{aligned}
$$
by

For PHS $h_{1}$ can be calculated analytically and is given

$$
\frac{h_{1} \beta}{\sigma_{c}}=\phi_{p}^{r}\left(1+\frac{q}{2}\right),
$$

while for EVI-polymers it depends not only on $\Delta$, but also on the depletion thickness near a wall, $\Delta_{w}$. This can be found from (5) by taking the limit $q \rightarrow 0$. In Fig. 5 we plot $h_{1}$ as a function of polymer concentration and show that in case of ideal polymers (dotted line + symbol) this term rises faster than in case of EVI-polymers (full line + symbol). The next term, $g$, depends on the triple overlap volume [volume 3 in Fig. 1(b)] and is more difficult to calculate

$$
\begin{aligned}
g & =-\int d z \int d \mathbf{r} U_{3} \\
& =\int d z \int d \mathbf{r} \int_{0}^{n_{p}^{r}} d n_{p}^{\prime}\left(\frac{\partial \Pi}{\partial n_{p}^{\prime}}\right) V_{o} \\
& =\int_{0}^{n_{p}^{r}} d n_{p}^{\prime}\left(\frac{\partial \Pi}{\partial n_{p}^{\prime}}\right) \int d z \int d \mathbf{r} V_{o} .
\end{aligned}
$$

The resulting geometrical problem is closely related to problems described by Bellemans ${ }^{33}$ and Fischer. ${ }^{34}$ There, however, the problem concerns two hard particles at a hard wall, while here we have soft particles at a wall which breaks some of the symmetry and changes the limits of integration.

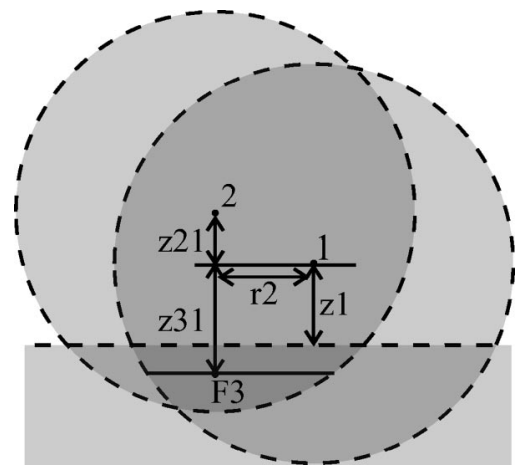

FIG. 6. Explanation of the notation used in (19) following Fischer (Ref. 34).

We follow the approach and notation of Fischer, change to cylindrical coordinates around particle 1 [shown in Fig. 6) and write the integrals in (18)] as

$$
\int d z \int d \mathbf{r} V_{o}=2 \pi \int d z_{1} \int d z_{21} \int d z_{31} \int d r_{2} r_{2} F_{3},
$$

with $F_{3}$ the overlap area between two disks at height $z_{31}$. For EVI-polymers and general $q$ (19) can readily be calculated numerically, while for PHS and the symmetric case of $q=1$ the integrals can be rewritten to much simpler integrals and (19) becomes $\frac{9}{280} \pi^{2} \sigma_{c}^{7}$ (see the Appendix), and $g$ reads

$$
\frac{g \beta}{\sigma_{c}^{4}}=\frac{9}{280} \pi^{2} \sigma_{c}^{3} n_{p}^{r}=\frac{27}{140} \pi \phi_{p}^{r} .
$$

In Fig. 5 we plot $g$ as a function of polymer concentration. Again, in case of ideal polymers (dotted line) this term rises faster than in case of EVI-polymers (full line). Moreover, for EVI-polymers $g$ rapidly does not change anymore as a function of polymer concentration. The consequences of this for wetting will be discussed at the end of this section.

We now have all the ingredients of (16). Minimizing the interfacial tension with respect to $\rho$ gives rise to the EulerLagrange equation

$$
2 m \frac{d^{2} \rho}{d z^{2}}=\frac{d f(\rho)}{d \rho}-\mu_{c}
$$

with boundary condition

$$
-h_{1}+g \rho_{1}=\left.2 m \frac{d \rho}{d z}\right|_{z=0} .
$$

The boundary condition appears because of the wall and one has to solve

$$
h_{1}-g \rho_{1}=2 \sqrt{m\left(f\left(\rho_{1}\right)-\mu_{c} \rho_{1}+p_{c}\right)},
$$

which can be done graphically. ${ }^{24}$ At the wetting transition the three interfacial tensions in play satisfy $\gamma_{G S}=\gamma_{L S}+\gamma_{G L}$. This means that the areas B and C from Fig. 3 must be equal, since liquid at the wall (indicated by the arrow in Fig. 3 with a density higher than the bulk liquid density) costs more inhomogeneities (area B) which are balanced by a favorable interaction with the wall (area C).

For mixtures slightly off-coexistence in the gas phase the prewetting line can be calculated in the same way as the 
(a)
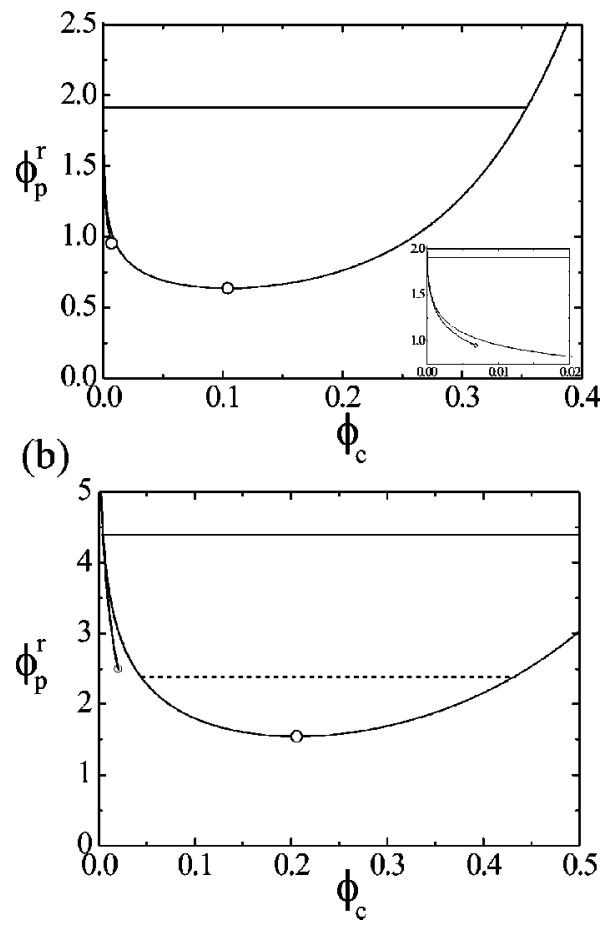

FIG. 7. Wetting phase diagrams in polymer reservoir representation with $q=1$ both for (a) ideal and (b) EVI-polymers. The full horizontal lines denote the location of the wetting transition. Mixtures are partially wetting above and completely wetting below these lines. The dashed line in (b) depicts the location of the triple line (crystal-fluid coexistence is not shown for the sake of clarity). The lines at the left/gas side of the phase diagrams represent the prewetting line starting from the wetting transition and ending in the prewetting critical point. The inset in (a) shows a zoom in on this area. The open circles depict critical points.

wetting transition. ${ }^{24}$ Now, only one true minimum appears indicating the density of the gas phase and one local minimum indicating the density of the metastable liquid phase. Again, an equal area construction can be made to determine the location of the prewetting line which starts at the wetting transition and ends in a prewetting critical point. ${ }^{35}$

In Fig. 7 we show the theoretical phase diagram in polymer reservoir representation for $q=1.0$ for (a) ideal polymers and (b) EVI-polymers. For ideal polymers a first-order wetting transition is found at $\phi_{p, w}^{r}=1.917$ with a prewetting line ending in a prewetting critical point. The wetting transition takes place rather close to the critical point; for comparison the triple line is at $\phi_{p, t}^{r}=6.081$. The triple line is found by the crossing of the gas-liquid and the fluid-crystal coexistence (calculated as described in Sec. II). In Ref. 10 the wetting transition is found at $\phi_{p, w}^{r}=0.85$, while in Ref. 12 it is found at $\phi_{p, w}^{r}=1.05$. Clearly, we find the transition further away from the critical point than in Refs. 10 and 12, but it is still much closer to the critical point than to the triple line. Furthermore, we calculate a prewetting line very close to the binodal whereas in Refs. 10 and 12 a first-order wetting transition was found, but not the accompanying prewetting line. We also find that increasing $h_{1}$ drives the wetting transition away from the critical point, while increasing $g$ counteracts this effect. Of course, higher-order terms in the contact density are present for the relatively large size ratio $q=1$, where mutual overlapping depletion zones of three and more particles are likely to occur, and taking these into account would even give better agreement with the predictions in Refs. 10 and 12.

In contrast, for EVI-polymers higher order terms contribute little. For example, for EVI-polymers the $g$-term does not change anymore with increasing polymer concentration from the bulk critical point on, see Fig. 5. Moreover, $g$ is very small compared to the result for ideal polymers $(0.050$ instead of $0.606 \times \phi_{p}^{r}$, Fig. 5). This means that the wetting transition for EVI-polymers is mainly driven by the $h_{1}$ term. The direct consequence of this is that the transition occurs far away from the critical point, at $\phi_{p, w}^{r}=4.403$ [indicated by the horizontal full line in Fig. 7(b)], which is even above the triple line, located at $\phi_{p, t}^{r}=2.388$ [indicated by the horizontal dashed line in Fig. 7(b)]. Note that in our calculations we can ignore the crystal phase and we are thus able to determine the location of the wetting transition even if it is above the triple line. Furthermore, the prewetting line is more extended than for ideal polymers and does not follow the binodal so closely. Wijting et al. ${ }^{14,15}$ find a wetting transition in a colloid-polymer mixture with $q=0.93$ and transform the experimental polymer concentration back to a theoretical reservoir polymer concentration thus locating the wetting transition between $4.3<\phi_{p, w}^{r}<4.5$. For $q=0.93$ we find the transition at $\phi_{p, w}^{r}=3.80$, which is again above the theoretical triple line. Yet in many experimental systems the crystalline phase seems to be supressed by colloidal sphere polydispersity.

\section{CONCLUSIONS}

We have calculated the interfacial tension of the free interface in phase separated colloid-polymer mixtures within a squared gradient approach. For ideal polymers we find reasonable agreement with a much more sophisticated approach. ${ }^{10}$ Moreover, by using a recently developed extension of the free volume theory for ideal polymers to excluded volume interacting polymers ${ }^{17}$ the polymer is incorporated much more realistically into the theory and the predicted bulk phase behavior agrees better with experiment. This first step should be taken when one wants to compare the predicted interfacial tensions with experimental values. Making this comparison we see that the ideal polymer description overestimates, while the EVI-polymer description underestimates the experimental data. The two different methods used to measure the interfacial tension in exactly the same system do not agree completely showing that measuring such ultralow interfacial tensions is very difficult.

Furthermore, we have put the often used qualitative interpretation of wetting in colloid-polymer mixtures based on particle wall overlap volumes and hence microscopic interactions into an insightful, semiphenomenological, quantitative form within the Cahn-Fisher-Nakanishi formalism. This way we clearly see which terms drive the transition to or away from the critical point. For ideal polymers we find that the location of the wetting transition is somewhat further away from the critical point than predicted by the theory of Ref. 10 and by the computer simulations of Ref. 12, but still 
(a)

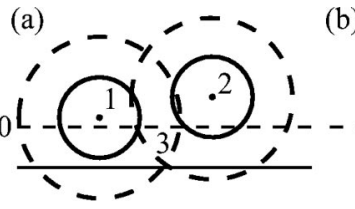

(b)

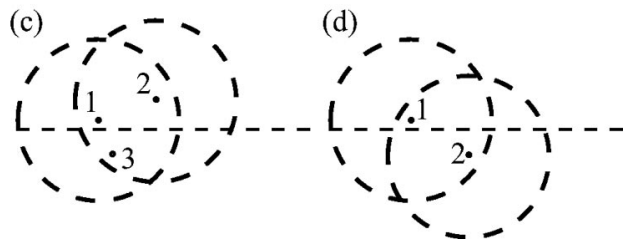

FIG. 8. Pictures to support the derivation given in the Appendix. Dashed lines mark depletion zones. The horizontal dashed line marks the depletion layer of the wall and particle-wall distances are relative to this line.

much closer to the critical point than to the triple line. We thus see that the two particle-wall term alone is not enough to drive the transition back to the critical point and that higher-order terms-although smaller-are important. Again, we can extend this model to incorporate EVIpolymers. In this case, the two-particle wall term is already very small, higher-order terms are even smaller and hence the wetting transition is driven away from the critical point by the attractive particle-wall term even to above the triple line. In experiment, however, a wetting transition is found, ${ }^{14,15}$ but in a system which does not display a crystal phase. We calculated the wetting transition for that system by ignoring the crystal phase. We then found a wetting transition reasonably close to the experimentally found one. Furthermore, in Ref. 15 it is mentioned that the pictures of the gas-liquid interface are somewhat fuzzy and we feel that experimentally the challenge still is in accurate measurements of the contact angle. Theoretically, the challenge is to describe polymers even more realistically in order to obtain not only a better overall agreement with the experimental bulk phase behavior, but also with the measured interfacial tensions.

\section{ACKNOWLEDGMENTS}

We thank Matthias Schmidt, Bob Evans, Joseph Indekeu, and Remco Tuinier for useful discussions. This work was supported by the Stichting voor Fundamenteel Onderzoek der Materie (Foundation for Fundamental Research on Matter), which is part of the Nederlandse Organisatie voor Wetenschappelijk Onderzoek (Netherlands Organization for Advancement of Research).

\section{APPENDIX: EQUATION (19) FOR $q=1$}

For $q=1$ we can calculate (19) analytically. First we calculate all volumes of type 3, see Fig. 8(a), as if particles 1 and 2 do not feel each other and next we subtract all volumes 3 where particles 1 and 2 did penetrate each other. For the first calculation we do not perform the calculation with $z_{1}$ as the axis of symmetry, but we choose the axis of symmetry around a ghost particle, particle 3 , at the other side of the depletion layer, see Fig. 8(b). If particles 1 and 2 lie within the shaded volume, $V_{s}$, in Fig. 8(b), then their depletion zones certainly overlap at the center of particle 3 . Doing this for all positions of particle 3 , with $z_{3}$ the distance of particle 3 to the depletion wall, gives the first part of the integration

$$
\begin{aligned}
\int_{0}^{\sigma_{c}} d z_{3} V_{s}^{2}\left(z_{3}\right) & =\int_{0}^{\sigma_{c}} d z_{3}\left(\frac{\pi}{3}\left(\sigma_{c}-z_{3}\right)^{2}\left(2 \sigma_{c}+z_{3}\right)\right)^{2} \\
& =\frac{11}{105} \pi^{2} \sigma_{c}^{7} .
\end{aligned}
$$

This is exactly the same calculation (in a different way) as done by Bellemans in Ref. 33.

The second part of the integration can be understood as follows. We let ghost particle 3 scan the volume behind the depletion wall, see Fig. 8(c), but therefore particle 3 should always be within a diameter $\sigma_{c}$ of particles 1 and 2, just as particle 1 should be within $\sigma_{c}$ of 2 and 3 , and 2 with respect to 1 and 3 . Thus, all particles are identical. One can easily see that if we put and keep particle 2 behind the wall as in Fig. 8(d) and let particle 3 scan the overlap volume between particles 1 and 2 behind the wall for all positions of 1 and 2 -let us call this integration $a$-that this integration is exactly the same as letting particle 3 scan before the wall again for all positions of particles 1 and 2, called integration $b$. Because of symmetry these integrations are exactly equal to integration $c$ with particles 1 and 2 before and particle 3 behind the wall, depicted in Fig. 8(c), which we want to know. We can easily do integrations $a$ and $b$ together since the total overlap volume between 1 and 2 is straightforward and $a+b$ becomes:

$$
\begin{aligned}
a+b & =\int_{0}^{\sigma_{c}} d z_{1} \int_{0}^{\sigma_{c}-z_{1}} d z_{2} V_{o}\left(z_{1}, z_{2}\right) 2 \pi\left(z_{1}+z_{2}\right) z_{2} \\
& =\frac{61}{420} \pi^{2} \sigma_{c}^{7},
\end{aligned}
$$

with $V_{o}\left(z_{1}, z_{2}\right)$ the overlap volume between particles 1 and $2, z_{x}$ the distance of particle $x$ to the depletion wall and $2 \pi\left(z_{1}+z_{2}\right) z_{2} d z_{2}$ the number of points 2 in a shell around particle 1. Bellemans calculated $a+b+c$ in Ref. 33 in a different way. Now, $c=\frac{1}{2}(a+b)$ and our integration (19) becomes

$$
\left(\frac{11}{105}-\frac{61}{840}\right) \pi^{2} \sigma_{c}^{7}=\frac{9}{280} \pi^{2} \sigma_{c}^{7} .
$$

${ }^{1}$ W. C. K. Poon, J. Phys.: Condens. Matter 14, R859 (2002).

${ }^{2}$ G. A. Vliegenthart and H. N. W. Lekkerkerker, Prog. Colloid Polym. Sci. 105, 27 (1997).

${ }^{3}$ E. H. A. de Hoog and H. N. W. Lekkerkerker, J. Phys. Chem. B 103, 5274 (1999).

${ }^{4}$ B. H. Chen, B. Payandeh, and M. Robert, Phys. Rev. E 62, 2369 (2000).

${ }^{5}$ A. Vrij, Phys. A. 235, 120 (1997).

${ }^{6}$ S. Asakura and F. Oosawa, J. Polym. Sci. 33, 183 (1958).

${ }^{7}$ A. Vrij, Pure Appl. Chem. 48, 471 (1976).

${ }^{8}$ J. M. Brader and R. Evans, Europhys. Lett. 49, 678 (2000).

${ }^{9}$ H. N. W. Lekkerkerker, W. C. K. Poon, P. N. Pusey, A. Stroobants, and P. B. Warren, Europhys. Lett. 20, 559 (1992).

${ }^{10}$ J. M. Brader, R. Evans, M. Schmidt, and H. Löwen, J. Phys.: Condens. Matter 14, L1 (2002).

${ }^{11}$ M. Schmidt, H. Löwen, J. M. Brader, and R. Evans, Phys. Rev. Lett. 85, 1934 (2000).

${ }^{12}$ M. Dijkstra and R. van Roij, Phys. Rev. Lett. 89, 208303 (2002). 
${ }^{13}$ D. G. A. L. Aarts, J. H. van der Wiel, and H. N. W. Lekkerkerker, J. Phys.: Condens. Matter 15, S245 (2002).

${ }^{14}$ W. K. Wijting, N. A. M. Besseling, and M. A. C. Stuart, Phys. Rev. Lett. 90, 196101 (2003)

${ }^{15}$ W. K. Wijting, N. A. M. Besseling, and M. A. C. Stuart, J. Phys. Chem. B 107, 10565 (2003).

${ }^{16}$ M. Fuchs and K. S. Schweizer, Europhys. Lett. 51, 621 (2000).

${ }^{17}$ D. G. A. L. Aarts, R. Tuinier, and H. N. W. Lekkerkerker, J. Phys.: Condens. Matter 14, 7551 (2002).

${ }^{18}$ M. Schmidt and M. Fuchs, J. Chem. Phys. 117, 6308 (2002).

${ }^{19}$ P. G. Bolhuis, A. A. Louis, and J. P. Hansen, Phys. Rev. Lett. 89, 128302 (2002).

${ }^{20}$ P. G. Bolhuis, A. A. Louis, and E. J. Meijer, Phys. Rev. Lett. 90, 068304 (2003).

${ }^{21}$ M. Schmidt, A. R. Denton, and J. M. Brader, J. Chem. Phys. 118, 1541 (2003).

${ }^{22}$ Y.-L. Chen, K. S. Schweizer, and M. Fuchs, J. Chem. Phys. 118, 3880 (2003).
${ }^{23}$ P. Paricaud, S. Varga, and G. Jackson, J. Chem. Phys. 118, 8525 (2003).

${ }^{24}$ J. W. Cahn, J. Chem. Phys. 66, 3667 (1977).

${ }^{25}$ H. Nakanishi and M. E. Fischer, Phys. Rev. Lett. 49, 1565 (1982).

${ }^{26}$ D. Bonn and D. Ross, Rep. Prog. Phys. 64, 1085 (2001).

${ }^{27}$ N. F. Carnahan and K. E. Starling, J. Phys. Chem. 51, 635 (1969).

${ }^{28}$ D. Frenkel and A. J. C. Ladd, J. Chem. Phys. 81, 3188 (1984).

${ }^{29}$ L. Schäfer, Excluded Volume Effects in Polymer Solutions (SpringerVerlag, Heidelberg, 1999).

${ }^{30}$ A. Hanke, E. Eisenriegler, and S. Dietrich, Phys. Rev. E 59, 6853 (1999).

${ }^{31}$ J. F. Joanny, L. Leibler, and P. G. De Gennes, J. Polym. Sci., Polym. Phys. Ed. 17, 1073 (1979).

${ }^{32}$ R. Tuinier, H. N. W. Lekkerkerker, and D. G. A. L. Aarts, Phys. Rev. E 65, 060801 (2002).

${ }^{33}$ A. Bellemans, Physica (Amsterdam) 28, 493 (1962).

${ }^{34}$ J. Fischer, Mol. Phys. 34, 1237 (1977).

${ }^{35}$ D. Bonn, H. Kellay, and J. Meunier, Phys. Rev. Lett. 73, 3560 (1994). 